\title{
Comment on the Paper: How to Intervene in the Caries Process: Early Childhood Caries - A Systematic Review by Schmoeckel et al.
}

\author{
Anju Varughese ${ }^{a}$ Venkitachalam Ramanarayanan ${ }^{b}$ Parvathy Balachandran $^{b}$ \\ Chandrashekar Janakiram $^{b}$ Induja Devan ${ }^{b}$ Vineetha Karuveettil ${ }^{b}$ \\ ${ }^{a}$ Department of Conservative Dentistry and Endodontics, Amrita School of Dentistry, Amrita Vishwa Vidyapeetham,

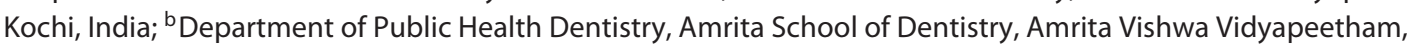 \\ Kochi, India
}

Dear Editor,

We write to you with reference to the article entitled "How to Intervene in the Caries Process: Early Childhood Caries - A Systematic Review” [Schmoeckel, 2020] published in your journal.

We found the article an interesting read and, in the process, had a few queries. We would appreciate if the same could be clarified by the authors.

In the PRISMA flow diagram (Fig. 1), we noticed a discrepancy in the numbers of articles included in various stages. The total number of records screened is mentioned as 148. However, we feel it had to be 158 (401-264 $+21=158)$. Subsequently, after removal of 100 articles after abstract screening, the remaining full text screening number should have been 58 instead of the mentioned 48 . There is also a lack of clarity in the studies not included in the last stage. We feel it should have been $25(11+6+$ 8 ). Kindly clarify as it is very confusing.

It is mentioned that 21 articles were retrieved from other sources. It would be beneficial if the authors had specified the source for replicability of literature search.

It is mentioned that quality assessment was done depending on the type of studies. Systematic reviews were assessed for quality using the PRISMA checklist while

karger@karger.com

(c) 2021 S. Karger AG, Basel

www.karger.com/cre

Karger non-randomized studies were assessed using the New Castle Ottawa scale in this study. But PRISMA is basically a reporting checklist and not a tool for assessing the risk of bias for systematic review. Validated tools like AMSTAR 2 [Shea et al., 2017] are available and could have been used here. Also, the Non-Operative Caries Management group contained randomized controlled trials (RCTs) and cohort studies, and we wonder how the New Castle Ottawa scale could have been used to assess the risk of bias of RCTs.

Finally, we also had concerns on the study design being called a systematic review as it has already included previously published systematic reviews and other study designs. Its neither purely a systematic review nor an overview of reviews.

Indeed, the article is an interesting read and gives a good overview on the evidence of management of early childhood caries. But overall, we feel that the methodological quality could have been improved.

\section{Conflict of Interest Statement}

The authors have no conflicts of interest to declare. 


\section{Funding Sources}

No funding was received.

\section{Author Contributions}

Anju Varughese: conception or design of the work; or the acquisition, analysis, or interpretation of data for the work; drafting the work; final approval of the version to be published; agreement to be accountable for all aspects of the work. Venkitachalam Ramanarayanan: analysis, or interpretation of data for the work; drafting the work; final approval of the version to be published; agreement to be accountable for all aspects of the work. Parvathy Balachandran: interpretation of data for the work; drafting the work; final approval of the version to be published; agreement to be accountable for all aspects of the work. Chandrashekar Janakiram: conception or design of the work; or the acquisition, analysis, or interpretation of data for the work; drafting the work; final approval of the version to be published; agreement to be accountable for all aspects of the work. Induja Devan: interpretation of data for the work; drafting the work; final approval of the version to be published; agreement to be accountable for all aspects of the work. Vineetha Karuveettil: interpretation of data for the work; drafting the work; final approval of the version to be published; agreement to be accountable for all aspects of the work.

References

Schmoeckel J, Gorseta K, Splieth CH, Juric H. How to Intervene in the Caries Process: Early Childhood Caries - A Systematic Review. Caries Res. 2020;54(2):102-12.

Shea BJ, Reeves BC, Wells G, Thuku M, Hamel C, Moran J, et al. AMSTAR 2: a critical appraisal tool for systematic reviews that include randomised or non-randomised studies of healthcare interventions, or both. BMJ (Clinical research ed). 2017 21;358:j4008. 